\title{
A Novel Paradigm for Computer-Aided Design: TRIZ-Based Hybridization of Topologically Optimized Density Distributions
}

\author{
A. Cardillo ${ }^{1}$, G. Cascini ${ }^{1}$, F.S. Frillici ${ }^{2}$, and F. Rotini ${ }^{2}$ \\ ${ }^{1}$ Dip. di Meccanica, Politecnico di Milano, Campus Bovisa Sud \\ via La Masa, 1-20156 Milano, Italy \\ ${ }^{2}$ Dip. di Meccanica e Tecnologie Industriali, Università di Firenze, \\ via di Santa Marta, 3-50139 Firenze, Italy \\ alessandro.cardillo@kaemart.it, gaetano.cascini@polimi.it, \\ \{francescosaverio.frillici, federico.rotini\}@unifi.it
}

\begin{abstract}
In a recent project the authors have proposed the adoption of Optimization Systems [1] as a bridging element between Computer-Aided Innovation (CAI) and PLM to identify geometrical contradictions [2], a particular case of the TRIZ physical contradiction [3]. A further development of the research [4] has revealed that the solutions obtained from several topological optimizations can be considered as elementary customized modeling features for a specific design task. The topology overcoming the arising geometrical contradiction can be obtained through a manipulation of the density distributions constituting the conflicting pair. Already two strategies of density combination have been identified as capable to solve geometrical contradictions and several others are under extended testing. The paper illustrates the most recent results of the ongoing research mainly related to the extension of the algorithms from 2D to 3D design spaces. The whole approach is clarified by means of two detailed examples, where the proposed technique is compared with classical multi-goal optimization.
\end{abstract}

Keywords: Computer-Aided Innovation, Computer-Aided Conceptual Design, Embodiment Design, Topological optimization, TRIZ.

\section{Introduction and Related Art}

Despite it is widely recognized the relative importance of conceptual design, due to its influential role in determining product's fundamental features, as a matter of facts, $\mathrm{CAD} / \mathrm{CAE}$ systems are not conceived to allow fast input and representation of concept models, and consequently they introduce inertial barriers in experimenting new models of design solutions. Indeed they don't provide any support to designers in developing and expressing their creativity $[5,6]$.

In fact, commercial CAD systems let the users successfully carry out tasks related to the detailed design stage, but not enough efforts have been dedicated to the conceptual design phase, especially activities such as function synthesis, concept generation and exploration. 
Preliminary attempts to provide conceptual design capabilities to CAD systems are in progress: in [7] shape and topological variations of a 3D model are proposed as a means to generate an optimal geometry through the application of genetic algorithms. Nevertheless, topological and shape variations are obtained through the modification of classical 3D modeling features, which dramatically limit the design space and impact the practical usability of the proposed method.

Topology Optimization is a technique that determines the optimal material distribution within a given design space, by modifying the apparent material density defined as design variable. The design domain is subdivided into finite elements and the optimization algorithm alters the material distribution within the design space at each iteration, according to the objective and constraints defined by the user. The surfaces defined as "functional" by the user, are preserved from the optimization process and considered as "frozen" areas by the algorithm.

Thus, designing through Topology Optimization technique means translating a design task into a mathematical problem with the following basic entities:

- An Objective Function, i.e. a combination of Evaluation Parameters, adopted as a reference metric to assess the degree of satisfaction of the design requirements;

- A set of Design Variables, i.e. material density variables by which the design domain is parameterized; they constitute the Control Parameters of the system affecting the Evaluation Parameters.

- A set of External Inputs and Constraints representing the operating conditions and requirements the system has to satisfy. Among them, manufacturing constraints may be set in order to take into account the requirements related to the manufacturing process. Sliding planes and preferred draw directions may be imposed for molded, tooled and stamped parts as well as minimum or maximum size of the structural elements (i.e. ribs, wall thicknesses, etc.).

The optimization algorithm finds the material density distribution within the given design domain which minimizes, maximizes, or, in general, "improves" the objective function, i.e. the Evaluation Parameters, while satisfying the Constraints.

Although Topology Optimization was born with the aim to support design tasks related to structural analyses, it has been recently applied to address design problems also in other fields such as: fluid dynamics, heat transfer and non linear structure behavior. Several works are available in literature, examples are provided in [8-10].

However, since the design process has multidisciplinary characteristics, improving one performance of a system may result in degrading another. This kind of conflicts cannot be solved using Design Optimization since these techniques are able to focus the design task only to one specific performance to be improved. More precisely, Design Optimization tools allow to manage multiple goals just by defining complex objective functions where a weight must be assigned to each specific goal [11]. Thus, the best compromise solution is generated on the base of an initial assumption made by the designer about the relative importance of the requirements, without taking into account the reciprocal interactions.

The integration among Topology Optimization technique and CAD tools is another very important open issue that should be addressed in order to enhance interoperability. As stated so far, Topology Optimization uses a material density distribution within a given design domain to represent a geometry: this paradigm cannot be directly translated into the feature-based representation used in CAD tools. 
In [4] the authors have presented an approach for hybridization of optimized density distributions based on TRIZ manipulation capable to overcome certain types of contradiction and they have illustrated the effectiveness of the proposed technique by means of two examples, the first related to the redesign of a motor-scooter wheel which should be manufactured using plastic material instead of aluminium alloy; the second related to the design of a linear guidance system that experiences two different loading conditions. Both the examples were related to $2 \mathrm{D}$ design problems. In this paper further developments of the research in progress are presented: extension of the density hybridization technique to a general 3D design space; application of the proposed approach also to geometrical contradictions obtained by the comparison of more than two conflicting boundary conditions. New examples have been developed to demonstrate the efficiency of the proposed approach and with this purpose a comparison between traditional multi-goal optimization, genetic algorithms applied to topological optimization and the TRIZ-based hybridization technique are presented. Section 2 summarizes the theoretical foundation of the suggested approach, while the hybridization algorithm is detailed in section 3. In Section 4 two applications of the method to 2D and 3D case studies are presented and discussed.

\section{A New Role for Topology Optimization Systems}

Innovation and optimization are usually conceived as conflicting activities. Besides, topology and shape generation capabilities of modern design optimization technologies can be adopted as a means to speed-up the embodiment of innovative concepts, but also as a way to support the designer in the analysis of conflicting requirements for an easier implementation of TRIZ instruments for conceptual design. In facts: (i) defining a single multi-goal optimization problem leads to a compromise solution; (ii) besides, defining $\mathrm{N}$ complementary mono-goal optimization problems, each with specific boundary conditions, leads to $\mathrm{N}$ different solutions; (iii) these solutions can be conflicting and this is the key to find contradictions.

In [1] it was proposed a classification of these contradictions mostly related to the geometrical differences between the results of the mono-goal optimization tasks and to the nature of the conflicting design parameters.

Instead of accepting a compromise solution generated by a multi-goal optimization, it is preferable to determine the best geometry for each boundary condition the technical system may encounter and, if these results conflict each other, adopt a TRIZ approach to overcome the emerging contradictions.

In [4] the authors formalized the concept of contradiction from Classical TRIZ in the case of geometrical contradiction identified by the comparison of topological optimizations. The minimal contradiction involves two alternative density distributions arising from two topological optimizations of the same technical system (TS) where different boundary conditions are applied, as schematically represented in figure 1: the symbols "+" and "-" mean that the behavior of the TS under the i-th Boundary Condition improves and worsens respectively according to the goal function of the optimization problem. In other words, the diagram in figure 1 should be read as follows: the density distribution should assume the topology " $\wedge$ " in order to improve the behavior of the TS under the Boundary Condition \#1, but then it degrades the behavior under 


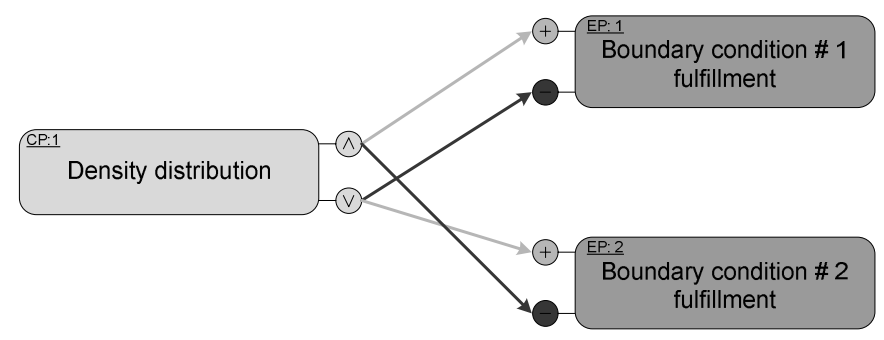

Fig. 1. Geometrical contradiction derived by the comparison of two topological optimizations related to alternative boundary conditions of the technical system. The density distribution is not a scalar variable, but a 3D-array representing the optimized density of each voxel.

Boundary Condition \#2 and should assume the topology " $\vee$ " in order to improve the behavior of the TS under the Boundary Condition \#2, but then it degrades the behavior under Boundary Condition \#1.

More generally a TS can experience more than two different operating conditions and consequently more than two topologically optimized density distributions can impact the same contradiction. The properties of such a "generalized contradiction" are still under investigation as well as the most effective directions to generate a satisfactory solution [12].

In [4] the authors have proposed the density distributions generated by topological optimizations of mono-goal problems as elementary customized feature for the definition of the geometry of a certain mechanical part during the embodiment stage, when its functional role must be translated into a geometry to be manufactured and coupled with other subsystems. The result of a topological optimization is a distribution of density so that each cell of the design space assumes a fuzzy value between 0 and 1 , which in turns means that boundaries are not rigid as it happens also with classical free-form modeling features; in facts, a density distribution can produce both topological and shape variations while, apart few exceptions, parametric modifications of a free-form surface produce just shape variations; compared with free-form surfaces where a shape variation is obtained by moving many control nodes, the output of a topological optimization produces different specific geometries by editing just one parameter, i.e. the threshold value of the density discriminating between void and filled space.

\section{The Hybridization Algorithm}

The investigation carried out by the authors about many different geometrical contradictions and related solutions revealed that typical solution paths can be associated to:

- different orientation of a geometrical feature, i.e. a rotation of a geometrical element, or in TRIZ terms, "Another Dimension" (Inventive Principle \#17);

- multiple copies obtained by a translation of a geometrical feature, as suggested from the trend of evolution Mono-Bi-Poly of homogeneous systems applied to geometrical features; 
- a combination of the above, i.e. the trend Mono-Bi-Poly applied to systems with shifted characteristics obtained by introducing multiple copies of a geometrical feature, each with a proper position and orientation; the simplest case is obtained by duplicating a geometrical feature by means of a mirror operation.

A general expression capable to represent all the above solution strategies is:

$$
\rho(x, y, z)=\frac{\sum_{i=1}^{N} \sum_{j=1}^{M i} K_{i j} \rho_{i}\left([R O T]_{i j}(x, y, z)^{T}+\left(x_{0}, y_{0}, z_{0}\right)_{i j}^{T}\right)}{\sum_{i=1}^{N} \sum_{j=1}^{M i} K_{i j}}
$$

where:

- $\mathrm{N}$ is the overall number of conflicting mono-goal optimizations (two if a classical TRIZ contradiction model is adopted);

- Mi is the number of "copies" of the i-th solution (step of a mono-bi-poly trend);

- Kij is the weight assigned to the $\mathrm{j}$-th copy of the $\mathrm{i}$-th distribution of density;

- [ROT]ij is the rotation applied to the $\mathrm{j}$-th copy of the $\mathrm{i}$-th distribution of density;

- $(x 0, y 0, z 0) i j$ is the translation applied to the $\mathrm{j}$-th copy of the $\mathrm{i}$-th distribution of density.

Since (1) must be applied to volumes segmented into finite elements (FE) and not to a continuum, rotations and translations imply that, in a general case, FE meshes don't overlap (figure 2, left). Thus, the calculation of the resulting density must be accomplished on a new mesh built upon the information contained in each rototranslated FE mesh (it is worth to remember that the density is here represented as a scalar value assigned to each voxel of each mesh containing the results of a monogoal opimization). Such a new mesh should be fine enough to keep the detail level of each mono-goal optimization; at the same time, it can't be constituted by an excessive number of extremely small elements, since it would imply too cumbersome calculations. In facts, as clarified by the 2D example in figure 2 left, the superposition of even just two meshes means calculating the resulting density in a number of elements much higher than the original size of the meshes themselves. Clearly, such an increase of the number of FE worsens when a 3D superposition is accomplished and even more when more than 2 meshes are combined.

In order to overcome such a limitation, it is proposed to adopt a single reference mesh characterized by elements keeping the same size of the original FE models; then, the density of each mono-goal optimization is projected onto the reference mesh through the creation of a number of virtual points. As shown in figure 2 right, the density of each FE is temporarily associated to a set of $n$ representative points homogeneously distributed in each voxel. Then, the density of each FE of the reference mesh (thick line) is estimated as the average of the density values associated to each representative-point contained within the limits of the FE itself.

A more detailed discussion about the relationship between the optimal value for the parameter $n$ and the size of the reference mesh is out of the scopes of the present paper and it is postponed to an upcoming publication. 


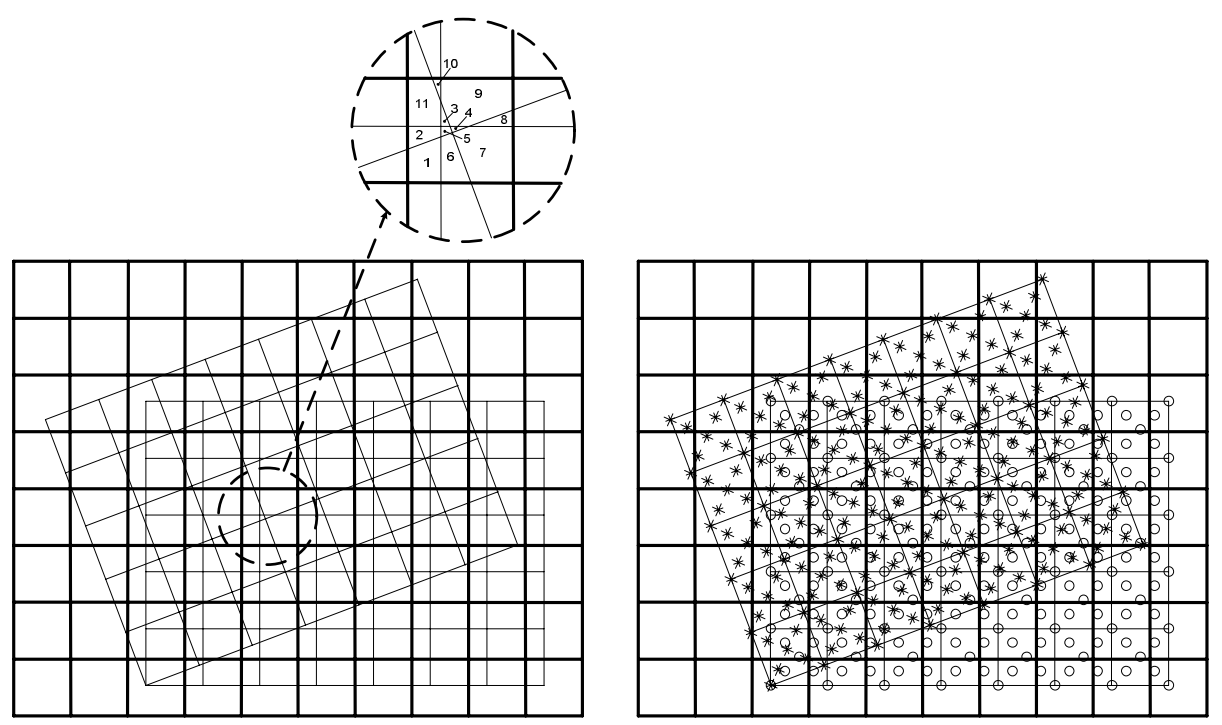

Fig. 2. Left: exemplary superposition of $2 \mathrm{D}$ rectangular meshes and consequent multiplication of the areas where a different combination of density should be applied. Right: the proposed technique consists in projecting on a reference mesh (thick line) the density resulting from each mono-goal optimization through a number of mass-points associated to each FE (here, $n=5$ ).

Once that each roto-translated density distribution of the mono-goal optimizations has been projected on the reference mesh, the hybridization formula (1) can be applied directly and a traditional threshold criterion can be adopted to remove the voxels having a lower density.

\section{Exemplary Applications}

In this section the proposed hybridization method is applied to:

- a plate under two conflicting load cases;

- a "three spokes" wheel undergoing three different operating conditions;

For each of the above mentioned case studies, a comparison among the hybridized topology and those obtained through a traditional multi-objective optimization process, is presented with the aim to assess the effectiveness of the proposed approach. The multi-objective traditional optimizations, as well as each mono-goal optimization, have been carried-out using the topology optimization code Optistruct rel. 8.0 by Altair [13].

\subsection{Plate under Two Conflicting Load Cases}

The first example is a trivial application to clarify the working principle and the potentialities of the proposed algorithm. The problem has been taken from [14] where the multi-objective topology optimization has been performed by means of Genetic 
Algorithms (GAs). It concerns the design of a plate having an overall dimension of $400 \times 300 \mathrm{~mm}$ and a thickness of $10 \mathrm{~mm}$, that experiences two different operating conditions, each constituted by a load having a magnitude of $200 \mathrm{~N}$ (figure 3). The plate is made of a steel alloy (elastic modulus $210 \mathrm{GPa}$ ).

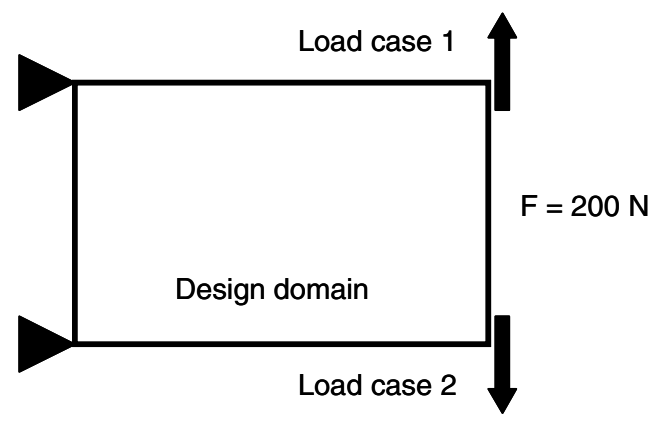

Fig. 3. Plate under two different load conditions [14]. The plate is fully constrained at the corners on the left edge and the forces are alternatively applied on the upper and lower corner of the right edge.

The optimization task consists in finding the optimal material distribution that minimizes the deformation energy of the plate, under a maximum mass constraint of 3 $\mathrm{kg}$. According to the proposed approach, two mono-objective optimizations have been performed under the same mass constraint: one for each load case. In figure 4 the resulting topologies are shown.
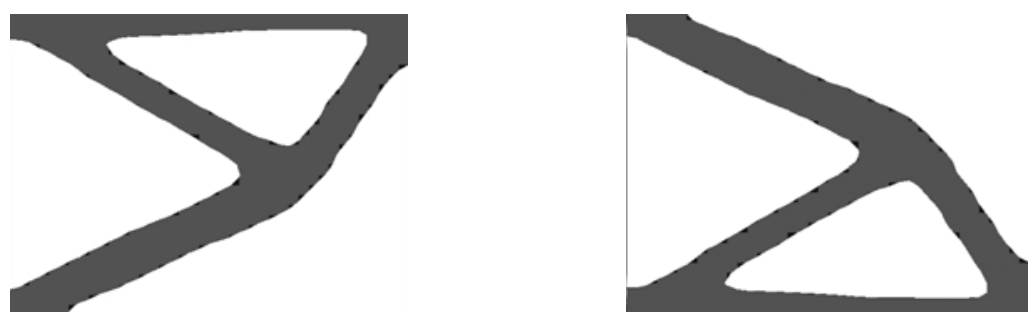

Fig. 4. Topologies resulting from the mono-objective optimizations: optimal density distributions under boundary conditions 1 (ledt) and 2 (right). The objective function is the minimal deformation energy under a maximum mass constraint of $3 \mathrm{~kg}$.

According to the hybridization formula, under the hypothesis of no repetitions $\left(\mathrm{M}_{\mathrm{i}}=1\right)$, and without any translation/rotation applied, the final density should be determined as the sum of the two mono-objective topologies presented in figure 3 . Each density distribution has been weighted with a coefficient equal to 1 , this is in accordance with the magnitude of the deformation energy that is the same for both the load cases. The hybrid topology obtained under these hypotheses is shown in figure 4 . This shape has been obtained by soiling the density distribution according to the mass constraint of $3 \mathrm{~kg}$. 
As a comparison means, a traditional multi-objective optimization has been accomplished by assigning the same relevance to the two objectives, i.e. considering as objective function the sum of the deformation energy of each load case. In figure 5 the obtained topology is shown, it has been soiled with a proper density threshold value in order to meet the mass constraint.
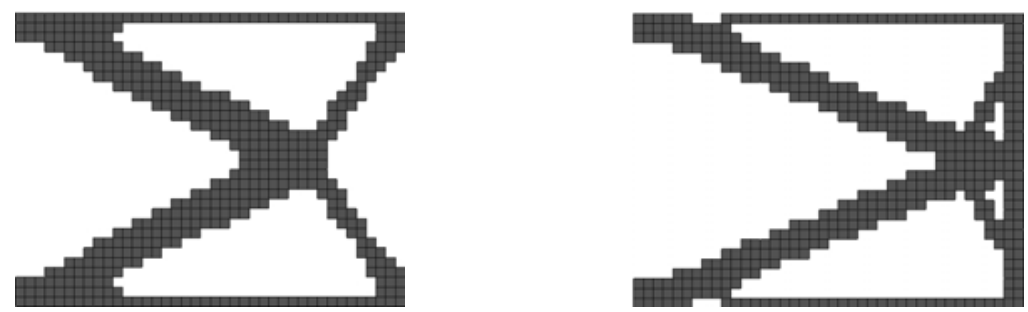

Fig. 5. Comparison between the topology obtained through the application of the proposed hybridization approach (left) and the result of the multi-goal optimization with balanced weight of the load cases (right). Both the solution share the same mass constraint (maximum $3 \mathrm{~kg}$ ).

A benchmark among the solutions presented in figure 5 has been performed by evaluating the deformation energy under the two load cases experienced by the plate. The obtained results are shown in Table 1: the hybrid solution, indeed quite different from the solution obtained through the multi-objective optimization, reveals higher mechanical performances.

Table 1. Hybrid solution vs. Multi-Objective, deformation energy comparison for load case 1 and 2

\begin{tabular}{lc}
\hline \multicolumn{1}{c}{ Topology } & Deformation Energy $(\mathrm{mJ})$ \\
\hline 1 - Multi-Objective & 5.92 \\
2 - Hybrid & 5.21 \\
$\Delta(2$ vs. 1$)$ & $-12 \%$ \\
\hline
\end{tabular}

As mentioned above, this exemplary case study has been taken from [14], where it was used by the authors to assess the effectiveness of a multi-objective topology optimization method based on Genetic Algorithms (GAs). It is worth to notice that in this case, the hybridization approach brings to the same solution obtained by the GAs. Besides, in [4] GAs required an initial population of 200 different topologies and the optimal solution was obtained after 600 generations, while the hybridization method requires a much lower number of individuals, i.e. the number of mono-objective optimizations.

Thus, the proposed hybridization approach seems to be more efficient than GAs from the computational point of view, even if further investigations are required in order to check its robustness in terms of search of the global minima.

\subsection{Three Spokes Wheel}

The second case study is referred to the application of the hybridization method to a three spokes wheel, in order to improve its performances under three different load 
conditions. In such a case, the design domain is represented by a 3D design space; the load cases, that the wheel encounters during its real operating conditions, can be considered as a combination of the followings:

- Load case 1: a load acting in the radial direction, approximately constituted by half the weight of the scooter plus passenger, typical of the vehicle running at the cruise speed;

- Load case 2: a load having a direction parallel to the axis of the hub, representing the load acting on a curving vehicle;

- Load case 3: a load applied in a tangential direction, typical of binding breaking actions.

All these loads have been applied on each side of the rim channel in which the tyre is placed. In order to avoid concentrated loads, they have been distributed on four nodes of the mesh, on each side of the rim. The rim channel and the hub of the wheel have been defined as functional surfaces in the design domain, the displacements of the surface of the hub have been fully constrained along the radial, tangential and axial directions. For each of the three load a reference magnitude of $1000 \mathrm{~N}$ has been considered.

The objective of the optimization task was finding the best design which minimize the deformation energy under the three different load conditions. The material by which the wheel is made is ABS, having a charge of $30 \%$ in mass of glass fibres (elastic modulus around $20 \mathrm{GPa}$ ). The constraints of the optimization task were:

1. mass of the wheel $<3.3 \mathrm{~kg}$;

2. axial draw direction in order to guarantee the manufacturability through an injection molding process and a three spokes configuration;

According to these constraint the hybridization process has been performed taking into account the following mono-objective optimizations:

1. wheel under radial load;

2. wheel under axial load;

3. wheel under axial load acting in the opposite direction of condition 2;

4. wheel under tangential load;

The topologies resulting from conditions 2 and 3 were the same due to the manufacturing constraint imposed for the material. The geometries shown in the figure 5 are those obtained after the application of a threshold to meet the mass constraint of $3.3 \mathrm{~kg}$. The roughness of the resulting shapes is due to the direct translation of the soiled density distributions into a faceted STL model, obtained by means of the OSSMOOTH tool by Altair Hypermesh rev. 8.0.

In terms of generalized contradiction [12], the results summarized in figure 6 can be expressed in the following form: the array of density associated to each voxel of the design domain should assume the value producing topology a) in order to satisfy the requirements of load case 1 , and should assume the value producing topology b) according to the requirements of load case 2 , and should finally assume the value producing topology c) to satisfy the requirements of load case 4 . 

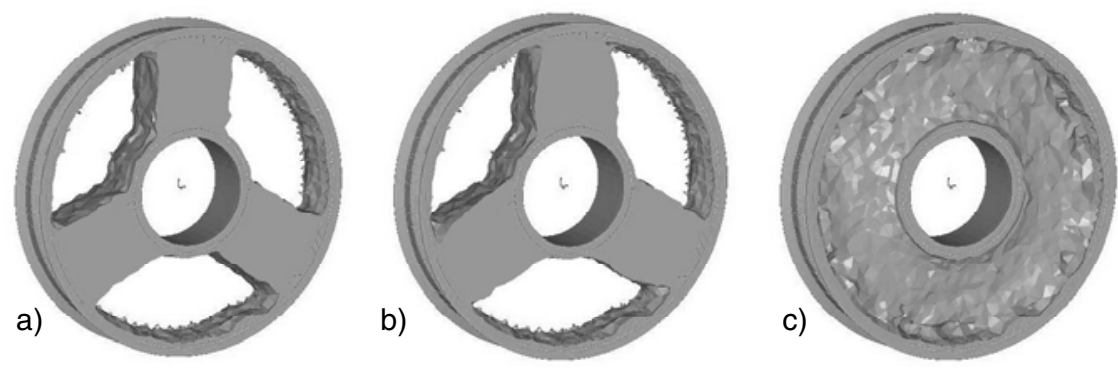

Fig. 6. Topologies resulting from three mono-goal optimization tasks. from left to right, under radial load (a), axial load (b) and tangential load (c). In such a case, radial and axial conditions produce quite similar topologies, despite some differences in the axial direction can be osserved, while the tangential load brings to a wheel having a continuous central rib. All these topologies meet the mass constraint of $3.3 \mathrm{~kg}$.

The hybridization formula (1) has been applied with the following values of the coefficients: $\mathrm{M}_{\mathrm{i}}=1, \mathrm{~K}_{\mathrm{i} 1}$ proportional to the overall deformation energy of each load case, no translations, nor rotations applied. In this case, the tangential and axial loads were more critical than the radial one. The results of the hybridization process are presented in figure 7, where two different relevant topologies are obtained by applying a different soiling threshold to the density distribution, both without violating the maximum mass constraint. A lighter version having three ribbed spokes and a weight of about $2.9 \mathrm{~kg}$ and another having three spokes with a continuous central rib and a weight of $3.2 \mathrm{~kg}$.

In order to perform the benchmark with a traditional multi-goal optimization process, the same design task has been performed with a multi-objective function. The density distribution has been soiled in order to meet the mass constraint; the topology shown in figure 7 (right) has a weight of about $3.2 \mathrm{~kg}$.
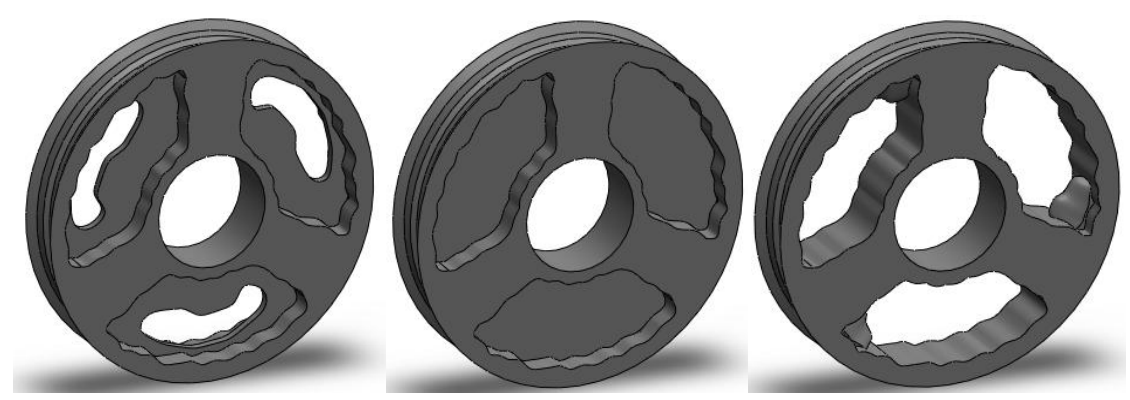

Fig. 7. Comparison between hybrid and multi-goal optimized topologies: from left to right, lighter version of the hybrid solution $(2.9 \mathrm{~kg})$, hybrid solution $(3.2 \mathrm{~kg})$, multi-objective $(3.2 \mathrm{~kg})$

A comparison among these topologies shows that the solution obtained with the traditional multi-objective optimization has the dimensions of the transversal section of the spokes larger than the spokes of the hybrid solution. Also the regions under the rim and the hub of the multi-objective solution are thicker than the hybrid ones. 
Table 2. Hybrid lighter vs. Multi-Objective and Hybrid heavier vs Multi-objective, comparison of the deformation energy $(\mathrm{mJ})$ for the three load cases

\begin{tabular}{lccc}
\hline \multicolumn{1}{c}{ Topology } & Load case 1 & Load case 2 & Load case 3 \\
\hline 1 - Multi-Objective & $0.97 \mathrm{E}-6$ & $1.18 \mathrm{E}-5$ & $7.35 \mathrm{E}-6$ \\
2 - Hybrid lighter & $0.96 \mathrm{E}-6$ & $1.15 \mathrm{E}-5$ & $5.33 \mathrm{E}-6$ \\
3 - Hybrid heavier & $0.96 \mathrm{E}-6$ & $1.15 \mathrm{E}-5$ & $3.96 \mathrm{E}-6$ \\
$\Delta(2$ vs. 1$)$ & $=$ & $=$ & $-27.5 \%$ \\
$\Delta(3$ vs. 1$)$ & $=$ & $=$ & $-46.1 \%$ \\
\hline
\end{tabular}

These differences in the material distribution bring a strong improvement of the behavior of the hybrid wheels (both lighter and heavier topologies) under the tangential load with respect to the multi-objective solution as shown in Table 2, while the other performances were essentially the same.

A critical issue for spoke wheels is represented by fatigue phenomena arising when the radial load changes position from the stiffer regions of the rim, such as those under the spokes, to the regions among the spokes. With the aim to evaluate the behavior of the hybrid heavier solution and the multi-objective under a rotating radial load, a distributed load has been applied on a arc of the rim among two spokes and the deformation of the central axis of the rim has been evaluated in different positions. Figure 8 clearly highlights that the hybrid solution presents a stiffness distribution along the rim much more uniform than the multi-objective. This is an unexpected result of the hybridization technique to be further investigated in order to understand if and how it impacts other performances of the system not considered as objectives of the optimization task.

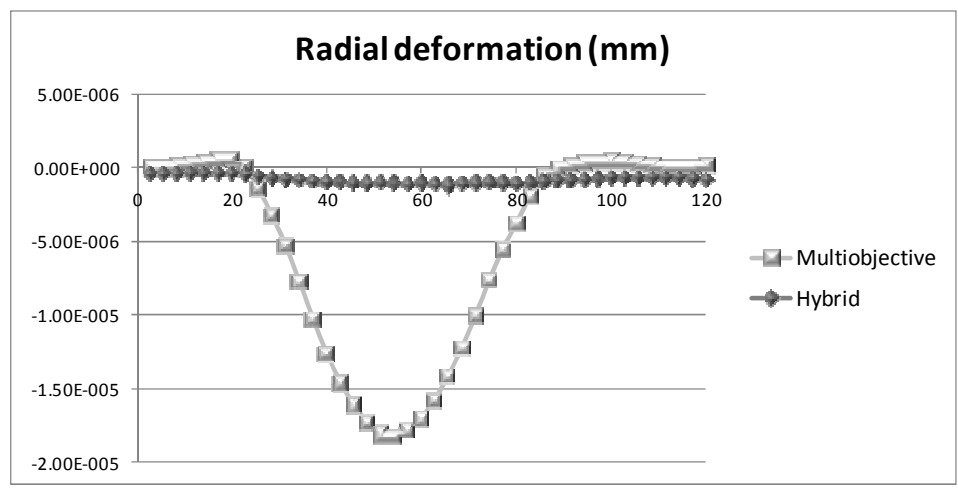

Fig. 8. Radial deformation among the spokes under a distributed radial loads acting on the wheel rim: comparison between hybrid and multi-objective optimization

\section{Discussion and Conclusion}

The examples reported so far are based on a straight sum of the density distributions obtained through the mono-goal optimizations. Besides, according to (1), the hybridization can be performed also by applying rotations, translations and multiple 
copies. This application of the proposed approach is still under investigation, but relevant results have been already achieved in several design tasks, even if the criteria to identify the most appropriate set of parameters governing (1) are still under study.

In the mind of the authors, translation and rotation are hybridization operators that can be useful for example when the optimization task is characterized by time dependent boundary conditions; in the wheel case study, all the three forces change position during the rotation of the wheel itself. In order to improve the behavior under fatigue loads, a uniform stiffness distribution around the hub is preferred. Indeed, a relative rotation, around the wheel axis, between the topologies a) and b) of figure 6 , brings to the generation of a six spokes geometry, as shown in figure 9. Such a configuration leads to a strong improvement of the overall fatigue behavior of the wheel, even if it slightly violates the mass constraint.

In conclusions, the proposed approach is based on the refusal of a compromise topology obtained through a traditional multi-objective optimization: better results can be achieved by hybridizing the density distributions determined with mono-goal optimizations corresponding to the different boundary conditions acting on the system. As shown also in the exemplary applications of the present paper, the proposed approach leads to very different topologies with respect to the traditional design optimization; the resulting geometry has often better performance than an equivalent multi-objective optimization, presumably because local optima are avoided as it happens with genetic algorithms (but with reduced computational efforts). Further studies are in progress, with the aim of investigating the opportunities arising from more complex manipulations of the mono-goal optimized topologies.

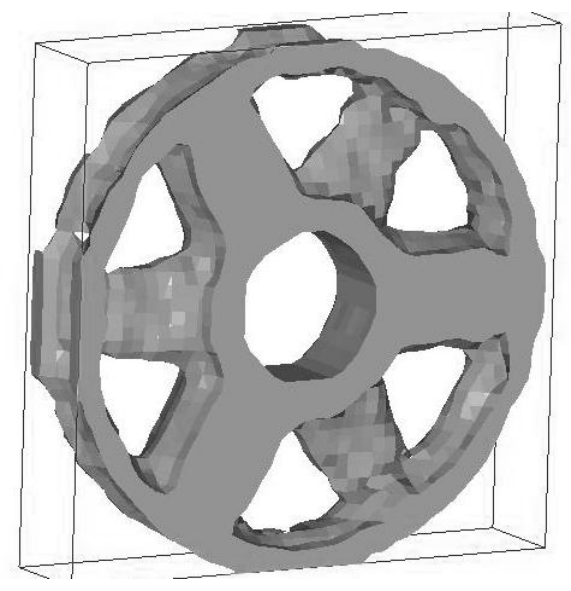

Fig. 9. Topology obtained through a hybridization based on the relative rotation of density distributions a) and b) of figure 5

\section{References}

1. Cascini, G., Rissone, P., Rotini, F.: From design optimization systems to geometrical contradictions. In: Proceedings of the 7th ETRIA TRIZ Future Conference, Frankfurt, Germany, November 6-8 (2007) 
2. Cugini, U., Cascini, G., Muzzupappa, M., et al.: Integrated Computer-Aided Innovation: the PROSIT approach. submitted for publications to the Special Issue on Computer-Aided Innovation of the Journal of Computers in Industry (2008)

3. Altshuller, G.S.: Creativity as an Exact Science: The Theory of the Solution of Inventive Problems. Gordon and Breach Science Publishers (1984) ISBN 0-677-21230-5 (original publication in Russian-1979)

4. Cascini, G., Cugini, U., Frillici, F., et al.: Computer-Aided Conceptual Design through TRIZ- based density manipulation. accepted for publication to the 19th CIRP Design Conference, Special session on Systematic Processes for Creative and Inventive Design, March 30-31. Cranfield University, UK (2009)

5. Qin, S.F., Harrison, R., West, A.A., et al.: A framework of web-based conceptual design. Computers in Industry 50(2), 153-164 (2003)

6. Tovey, M., Owen, J.: Sketching and direct CAD modeling in Automotive Design. Design Studies 21(6), 569-588 (2000)

7. Leon-Rovira, N., Cueva, J.M., Silva, D., et al.: Automatic shape and topology variations in 3D CAD environments for genetic optimization. International Journal of Computer Applications in Technology 30(1/2), 59-68 (2007)

8. Hutabarat, W., Parks, G.T., Jarret, J.P., et al.: Aerodynamic Topology Optimisation Using an Implicit Representation and a Multiobjective Genetic Algorithm. In: Monmarché, N., Talbi, E.-G., Collet, P., Schoenauer, M., Lutton, E. (eds.) EA 2007. LNCS, vol. 4926, pp. 148-159. Springer, Heidelberg (2008)

9. Bruns, T.E.: Topology optimization of convection-dominated, steady-state heat transfer problems. International Journal of Heat and Mass Transfer 50(15-16), 2859-2873 (2007)

10. Bruns, T.E., Tortorelli, D.A.: Topology optimization of non-linear elastic structures and compliant mechanisms. Computer Methods in Applied Mechanics and Engineering 190(26-27), 3443-3459 (2001)

11. Spath, D., Neithardt, W., Bangert, C.: Optimized design with topology and shape optimization. Proceedings of the Institution of Mechanical Engineers, Part B: Journal of Engineering Manufacture 216(8), 1187-1191 (2002)

12. Dubois, S., Rasovska, I., De Guio, R.: Comparison of non solvable problem solving principles issued from CSP and TRIZ. In: Proceedings of the 2nd Topical Session on Computer-Aided Innovation, 20th IFIP World Computer Congress, Milan, Italy, published on "Computers-Aided Innovation", September 7-10. Springer, Heidelberg (2008) ISBN 978-0-387-09696-4

13. http://www.altair.com

14. Aguilar Madeira, J.F., Rodrigues, H., Pina, H.: Multi-Objective optimization of structures topology by genetic algorithms. Advances in Engineering Software 36, 21-28 (2005) 